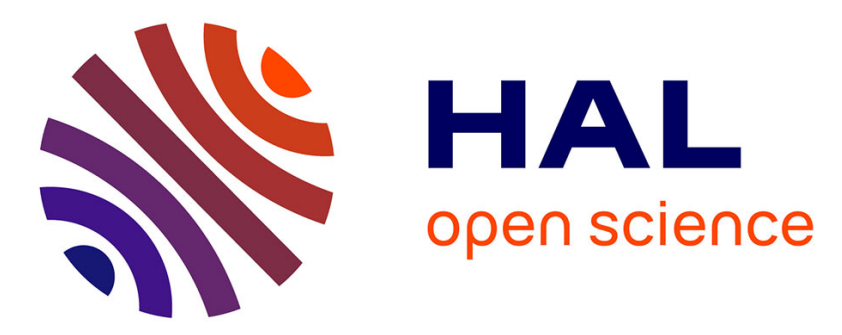

\title{
Etude de l'hétérosis chez le colza oléagineux d'hiver (Brassica napus L.). II. - Structure génétique d'une population de lignées
}

Marianne Lefort-Buson, Yvette Dattée

\section{To cite this version:}

Marianne Lefort-Buson, Yvette Dattée. Etude de l'hétérosis chez le colza oléagineux d'hiver (Brassica napus L.). II. - Structure génétique d'une population de lignées. Agronomie, 1985, 5 (3), pp.201-208. hal-00884751

\section{HAL Id: hal-00884751 \\ https://hal.science/hal-00884751}

Submitted on 1 Jan 1985

HAL is a multi-disciplinary open access archive for the deposit and dissemination of scientific research documents, whether they are published or not. The documents may come from teaching and research institutions in France or abroad, or from public or private research centers.
L'archive ouverte pluridisciplinaire HAL, est destinée au dépôt et à la diffusion de documents scientifiques de niveau recherche, publiés ou non, émanant des établissements d'enseignement et de recherche français ou étrangers, des laboratoires publics ou privés. 


\title{
Etude de l'hétérosis chez le colza oléagineux d'hiver (Brassica napus L.). II. - Structure génétique d'une population de lignées
}

\author{
Marianne LEFORT-BUSON \& Yvette DATTÉE $(*)$ \\ I.N.R.A., Station d'Amélioration des Plantes, Centre de Recherches de Rennes, B.P. 29, F 35650 Le Rheu \\ (*) I.N.R.A., Laboratoire d'Amélioration des Plantes, Université de Paris XI, F 91405 Orsay Cedex
}

RÉSUMÉ

\begin{abstract}
Ayant mis en évidence, dans un article précédent, un effet d'hétérosis chez le colza oléagineux d'hiver (Brassica napus L.), les auteurs s'intéressent ici à la décomposition de la variabilité et aux corrélations génétiques dans la population de lignées sélectionnées. Les résultats montrent, entre autres, une prépondérance de la variance d'aptitude générale à la combinaison (A.G.C.) dans l'expression de la variance génétique totale et une liaison positive entre valeur propre et A.G.C. des lignées parentales. Ils permettent aussi de discuter des orientations à donner à la sélection d'hybrides F1 et d'apporter une contribution à la connaissance de l'hétérosis.
\end{abstract}

Mots clés additionnels : Variabilité génétique, corrélations génétiques, sélection de variétés hybrides. population.

In a previous paper, the authors showed a heterosis effect for winter oilseed rape (Brassica napus L.) ; here, they are interested in the structure of variability and in the genetic correlations within a population of selfed lines. Two important results have been obtained : 1) most of the genetic variability can be explained by general combining ability (G.C.A.) ; 2) there is a positive correlation between the value of the parental selfed lines and their G.C.A. Based on the results obtained, the authors discuss the consequences for heterosis theory and way of identifying and developing superior inbreds for use in $\mathrm{Fl}$ hybrid rapeseed breeding.

Additional key words : Genetic variability, genetic correlations, selection of hybrid varieties.

\section{INTRODUCTION}

La première partie de l'étude a souligné l'intérêt économique de la production d'hybrides $\mathrm{F} 1$, chez le colza oléagineux d'hiver (Brassica napus L.) (LEFORTBUSON \& DATTEE, 1985). Il se pose alors le choix d'une méthode de sélection adaptée à ces nouvelles variétés.

L'efficacité d'une méthode de sélection est définie en termes de gain génétique par unité de temps ; or, pour un caractère donné, le gain génétique est fonction, entre autres, de la variance du caractère dans la population. De plus, le gain génétique pour un caractère peut être augmenté en utilisant l'information relative à d'autres caractères pour lesquels les corrélations génétiques avec le premier et les héritabilités sont élevées.

L'étude a donc porté à la fois sur l'évaluation et la comparaison des différentes sources de variabilité génétique et sur les corrélations génétiques entre caractères, dans la population considérée.

La variabilité génétique a été classiquement décomposée en aptitude générale à la combinaison (A.G.C.) et aptitude spécifique à la combinaison (A.S.C.), effets réciproques moyen et spécifique dans la population de lignées sélectionnées en 1979. En 1981, la même étude a été reprise sans tenir compte des effets réciproques.

\section{MATÉRIEL ET MÉTHODES}

\section{A. Dispositif expérimental}

L'étude simultanée des A.G.C. et des A.S.C. a été faite par un plan de croisement diallèle incomplet; ce plan est constitué de plusieurs diallèles élémentaires 
$(5 \times 5)$, respectivement 7 en 1979 et 6 en 1981. La structure du plan de croisement (nombre, $r$, et taille, $n$, des diallèles élémentaires) a été raisonnée pour obtenir une précision de la variance d'A.G.C. légèrement supérieure à celle de la variance d'A.S.C. (LEFORT, 1977) et en tenant compte des contraintes matérielles de production de semences hybrides.

En 1979, les 7 diallèles élémentaires complets ont conduit à 140 hybrides testés sur 2 répétitions. Les effets réciproques étudiés en 1979 étant rarement significatifs, leur étude n'a donc pas été reconduite en 1981 et il a été jugé préférable d'augmenter le nombre de répétitions du dispositif. Les 6 diallèles ont alors conduit à 60 hybrides testés sur 4 répétitions. Les parcelles d'essai comportent 4 (1979) ou 5 (1981) lignes de $3 \mathrm{~m}$ espacées de $0,3 \mathrm{~m}$; la densité de semis est de $4 \mathrm{~kg} / \mathrm{ha}$.

Les lignées parentales ont été choisies pour assurer une grande variabilité génétique et géographique, représentative de la population de lignées sélectionnées. Plusieurs lignées étrangères ont été utilisées : une par pays producteur au maximum ; elles ont été considérées comme non apparentées aux lignées françaises et non apparentées entre elles. Par ailleurs, la connaissance de la généalogie des lignées françaises a permis le calcul de leurs coefficients de parenté 2 à 2 (BUSON, 1979). Les lignées ont alors été réparties dans les diallèles élémentaires de façon que l'apparentement connu entre les géniteurs puisse être considéré comme nul dans chacun d'eux. Pour le dispositif dans son ensemble, par contre, la parenté n'est pas nulle. En 1979, une lignée sans acide érucique "Primor » utilisée dans chaque diallèle a permis d'établir un lien entre tous les dispositifs élémentaires.

\section{B. Caractères}

Les caractères étudiés, présentés dans la $1^{\text {re }}$ partie de l'article (LEFORT-BUSON \& DATTEE, 1985 ; tabl. 2), ne sont rappelés ici que pour mémoire. Il s'agit de la date de début floraison (DF), du couvert foliaire (CF), de la sensibilité à la verse (VERSE), du rendement en grains (RENDT), du poids de 1000 grains (P1000), des nombres (NG/SIL) et poids (PG/SIL) moyens de grains par silique. Dans le $1^{\text {er }}$ essai, le nombre de grains par silique a été mesuré sur la ramification principale (NG/SIL.RI) et globalement sur les ramifications secondaires (NG/SIL.RII). Pour diverses raisons, certains caractères n'ont pas été mesurés de la même manière en 1979 et en 1981 et d'autres n'ont pas été mesurés les 2 années.

\section{Méthode d'étude}

Dans le cas d'un diallèle complet, la valeur d'un hybride entre 2 lignées i et $\mathrm{j}$, mesuré dans le bloc $\mathrm{k}$ (dont l'effet est noté $b_{k}$ ), est décomposée selon le modèle (HAYMAN, 1954) :

$$
Y_{i j k}=\mu+G_{i}+G_{j}+M_{i}+M_{j}+S_{i j}+R_{i j}+b_{k}+E_{i j k}
$$

où :

- $\mathrm{G}_{\mathrm{i}}$ et $\mathrm{G}_{\mathrm{j}}$ sont les aptitudes générales à la combinaison des lignées $\mathrm{i}$ et $\mathrm{j}$;

- $\mathrm{M}_{\mathrm{i}}$ et $\mathrm{M}_{\mathrm{j}}$ sont les effets réciproques moyens des lignées $\mathrm{i}$ et $\mathrm{j}$;
- $\mathrm{S}_{\mathrm{ij}}$, l'aptitude spécifique à la combinaison du couple (ij) ; (ij) ;

- $\mathrm{R}_{\mathrm{ij}}$, l'effet réciproque spécifique lié au couple - $E_{i j k}$, une résiduelle d'espérance nulle.

Ces variables sont des aléatoires de variances respectives $\gamma_{g}, \gamma_{m}, \gamma_{s}, \gamma_{r}$ et $\gamma_{e}$. Du fait des contraintes de calcul informatique les variances n'ont été estimées qu'à partir de modèles à effets fixés.

\section{Estimations des variances génétiques}

Chaque diallèle élémentaire est analysé séparément et les résultats sont ensuite regroupés. Sous l'hypothèse d'homogénéité des variances génétiques des divers diallèles, les espérances des sommes de carrés relatives à chaque effet sont, en effet, égales quel que soit le diallèle ; leurs sommes constituent des estimations globales sans biais.

Par exemple, soit $S_{i}$ la statistique permettant d'estimer la variance d'A.S.C. dans le diallèle i :

$$
E\left(S_{i}\right)=\frac{n(n-3)}{2}\left(\gamma_{s}+\frac{\gamma_{\mathrm{ei}}}{2 b}\right)
$$

où $\gamma_{\mathrm{ei}}$ est la variance résiduelle associée au diallèle $\mathrm{i}$.

La statistique somme, $\mathrm{S}$, a pour espérance :

$$
E(S)=\frac{n(n-3)}{2} \sum_{i=1}^{r}\left(\gamma_{s}+\frac{\gamma_{e i}}{2 b}\right)
$$

ou

$$
\frac{\mathrm{m}(\mathrm{n}-3)}{2} \cdot\left(\gamma_{\mathrm{s}}+\gamma_{\mathrm{e}} / 2 \mathrm{~b}\right),
$$

en supposant l'égalité des variances résiduelles $\gamma_{\mathrm{ei}}$.

Un raisonnement identique s'applique pour les estimations de $\gamma_{\mathrm{g}}, \gamma_{\mathrm{m}}$ et $\gamma_{\mathrm{r}}$.

La précision de ces estimations ne peut être donnée exactement car les lois des statistiques $\mathrm{S}$ ne sont pas classiques. En effet, du fait de l'apparentement entre les lignées de diallèles différents, les sommes de carrés associées à chaque diallèle ne sont pas indépendantes entre elles.

Cependant, en utilisant en $1^{\text {re }}$ approximation les formules développées par LEFORT (1977), un intervalle de confiance approximatif de ces estimations a été calculé par les formules valables pour la loi normale asymptotique.

Le rapport $\hat{x}=2 \hat{\gamma}_{\mathrm{g}} /\left(2 \hat{\gamma}_{\mathrm{g}}+\hat{\gamma}_{\mathrm{s}}+\hat{\gamma}_{\mathrm{e}}\right)$ a été calculé : il correspond à la fraction de la variabilité transmise d'une génération à l'autre. En supposant les géniteurs consanguins et en l'absence d'épistasie, $\hat{\mathrm{x}}$ peut être assimilé à l'héritabilité $\left(\mathrm{h}^{2}\right)$ au sens strict.

\section{Liaisons génétiques}

Lorsque la majeure partie de la variance génétique est due aux effets d'A.G.C., ceux-ci ont été estimés à partir d'un modèle à effets fixés à défaut d'un modèle à effets aléatoires. Les estimations ne prennent alors en compte que l'information relative au caractère considéré et ne dépendent pas de la variance résiduelle, alors qu'estimées par le modèle à effets aléatoires, elles seraient fonction de l'information fournie par les autres caractères et de la précision de cette information. 
L'amplitude des différences entre les A.G.C. ainsi estimées devrait être égale ou supérieure à celle obtenue avec un modèle aléatoire ; cependant le classement des A.G.C. reste invariant car les dispositifs sont quasiment équilibrés. Par conséquent, le biais introduit par ce mode de calcul est peu important pour le classement des lignées.

Ont également été calculées :

- les corrélations entre A.G.C. des géniteurs pour les différents caractères ;

- les corrélations entre A.G.C. et valeur propre des lignées pour chaque caractère.

Par ailleurs, certains résultats déjà publiés en 1982 ont été mentionnés ici pour permettre une comparaison des 2 années d'essai.

\section{RÉSULTATS}

\section{A. Décomposition de la variabilité}

Les estimations des variances d'A.G.C. $\left(\hat{\gamma}_{g}\right)$ et d'A.S.C. $\left(\hat{\gamma}_{s}\right)$ sont présentées dans le tableau 1. Leur faible précision souligne les limites de l'étude. Des estimations plus précises de $\hat{\gamma}_{\mathrm{g}}$ et $\hat{\gamma}_{\mathrm{s}}$ auraient pu être obtenues soit en testant un plus grand nombre d'hybrides $\mathrm{F} 1$, soit en augmentant le nombre de répétitions du dispositif expérimental afin de réduire l'erreur résiduelle. Les contraintes matérielles liées à la production de semences hybrides par pollinisation manuelle ont limité l'extension du plan de croisement et du dispositif expérimental ; la concordance de certains résultats sur les 2 années d'expérimentation permet toutefois d'établir quelques conclusions.

Pour la majorité des caractères, les carrés moyens des A.G.C. sont supérieurs à ceux des A.S.C. (tabl. 1). Les effets d'aptitude à la combinaison spécifique sont non significatifs ou peu importants pour tous les caractères. Bien que ces derniers effets soient souvent non significatifs, il a paru intéressant de calculer le rapport $\hat{\gamma}_{\mathrm{g}} / \hat{\gamma}_{\mathrm{s}}$; celui-ci est nettement plus faible pour les nombres et poids de grains par silique que pour les autres caractères. Par ailleurs, à l'exception du rendement et du couvert foliaire, ce rapport est plus élevé en 1979 qu'en 1981. L'influence de l'année sur la variance génétique totale avait déjà été établie (LEFORT-BUSON \& DATTEE, 1985) ; le présent résultat montre de plus que l'effet de l'année se manifesterait de façon différente sur chaque composante de la variance génétique.

Pour les 2 années, et à l'exception de la date de début floraison et du poids de 1000 grains, le paramètre $\hat{x}$ est peu élevé (tabl. 1) : le gain génétique par cycle de sélection sera relativement limité pour chaque caractère. Malgré l'amplitude des intervalles de confiance sur les variances, l'homogénéité du paramètre $\hat{x}$ pour les 2 essais renforce les conclusions énoncées ci-dessus.

\section{B. Corrélations entre caractères}

A l'exception des corrélations entre le rendement et le couvert foliaire ainsi qu'entre les nombres et poids de grains par silique, les autres estimations des corrélations entre A.G.C. des géniteurs sont peu élevées $(\hat{\rho}<0,6)$. Quel que soit l'échantillonnage, les liaisons entre le rendement parcellaire et chacune des composantes sont faibles (tabl. 2). Bien que non significative et faible en valeur absolue, la liaison entre le rendement et le poids de 1000 grains est identique les 2 années $(\hat{\rho}=-0,16)$; elle pourrait traduire un effet compensateur du poids de 1000 grains dans l'élaboration du rendement, effet déjà mis en évidence au niveau phénotypique sur plantes individuelles (BUSON, 1979). A l'exception du nombre de grains par silique estimé sur la ramification principale, les corrélations

TABLEAU 1

Estimation des variances d'A.G.C. $\left(\hat{\gamma}_{g}\right)$, d'A.S.C. $\left(\hat{\hat{i}}_{s}\right)$, résiduelle $\left(\hat{\hat{i}}_{e}\right)$ et du rapport $\hat{x}=2 \hat{i}_{g} /\left(2 \hat{i}_{g}+\hat{i}_{s}+\hat{i}_{e}\right)$ dans les essais de 1979 (a) et 1981 (b).

Estimated variances of different effects : G.C.A. $\left(\hat{\gamma}_{g}\right)$, S.C.A. $\left(\hat{\gamma}_{s}\right)$, residuals $\left(\hat{\gamma}_{e}\right)$, and of the ratio $\hat{x}=2 \hat{\gamma}_{g} /\left(2 \hat{\gamma}_{g}+\hat{\gamma}_{s}+\hat{\gamma}_{e}\right)$ for both years, 1979 (a) and 1981 (b).

\begin{tabular}{|c|c|c|c|c|c|c|}
\hline \multicolumn{2}{|c|}{ Caractère } & $\hat{\gamma}_{\mathrm{g}}$ & $\hat{\gamma}_{\mathrm{s}}$ & $\hat{\gamma}_{\mathrm{g}} / \hat{\gamma}_{\mathrm{s}}$ & $\hat{\gamma}_{\mathrm{e}}$ & $\hat{\mathrm{x}}$ \\
\hline DF & (a) & $3,48 \pm 1,05$ & $0,23 \pm 0,61$ & 15,1 & 1,41 & 0,81 \\
\hline & (b) & $4,06 \pm 2,79$ & $0,57 \pm 0,59$ & 7,1 & 1,05 & 0,83 \\
\hline \multirow{2}{*}{$\mathrm{CF}$} & (a) & $0,201 \pm 0,145$ & $0,017 \pm 0,135$ & 11,8 & 0,956 & 0,29 \\
\hline & (b) & $0.0343+0.02 \times 5$ & $0,0032 \pm 0,0220$ & 10,7 & 0,2613 & 0,20 \\
\hline \multirow[t]{2}{*}{ RENDT } & (a) & $5,39 \pm 4,15$ & $0,32 \pm 3,55$ & 16,8 & 25,53 & 0,29 \\
\hline & (b) & $3,32 \pm 2,67$ & $0,21 \pm 1,95$ & 15,8 & 26,89 & 0,20 \\
\hline \multirow[t]{2}{*}{ P1000 } & (a) & $0,1373 \pm 0,0830$ & $0,0092 \pm 0,0202$ & 14,9 & 0,1838 & 0,59 \\
\hline & (b) & $0,023 \pm 0,015$ & $0,0023 \pm 0,0041$ & 10,0 & 0,0413 & 0,51 \\
\hline \multirow[t]{2}{*}{ VERSE } & (a) & $0,234 \pm 0,323$ & $0,015 \pm 0,698$ & 15,6 & 0,591 & 0,44 \\
\hline & (b) & - & - & - & - & - \\
\hline \multirow{2}{*}{ NG/SIL.RI } & (a) & $1,807 \pm 1,156$ & $0,424 \pm 0,857$ & 4,3 & 7,689 & 0,31 \\
\hline & (b) & - & - & - & - & - \\
\hline \multirow[t]{2}{*}{ NG/SIL.RII } & (a) & $0,483 \pm 0,507$ & $0,099 \pm 0,456$ & 4,9 & 4,508 & 0,17 \\
\hline & (b) & - & - & - & - & - \\
\hline \multirow[t]{2}{*}{ NG/SIL } & (a) & - & - & - & - & - \\
\hline & (b) & $0,813 \pm 0,723$ & $0,405 \pm 0,620$ & 2,0 & 5,924 & 0,20 \\
\hline \multirow[t]{2}{*}{$\mathrm{PG} / \mathrm{SIL}$} & (a) & - & - & - & - & - \\
\hline & (b) & $22,7 \pm 19,8$ & $13,7 \pm 16,5$ & 1,7 & 134,4 & 0,23 \\
\hline
\end{tabular}

N.B. Les estimations ponctuelles de $\gamma_{\mathrm{g}}$ et de $\gamma_{\mathrm{s}}$ sont accompagnées de leurs intervalles de confiances au niveau $1-\alpha=0,95$.

N.B. Estimates of $\gamma_{\mathrm{g}}$ and $\gamma_{\mathrm{s}}$ are followed by their confidence limit at $1-\alpha=0.95$. 
entre le rendement et chaque composante, une fois éliminé l'effet linéaire du poids de 1000 grains (corrélations partielles), sont légèrement supérieures aux corrélations simples associées. Ce résultat renforce l'hypothèse d'un effet compensateur du poids de 1000 grains.

La liaison entre le couvert foliaire au début floraison et le rendement parcellaire, estimée à partir des A.G.C., est élevée $(0,72)$; en revanche, l'estimation obtenue à partir des résidus est faible (LEFORT-BUSON \& DATTEE, 1985). L'hypothèse d'une liaison génétique entre ces 2 caractères peut être formulée ; elle est renforcée par le fait que chaque composante de la variance intervient de manière comparable pour les 2 caractères dans la variance génétique totale (voir tabl. 1). De plus, en 1979, les liaisons entre la résistance à la verse et, respectivement, le couvert foliaire et le rendement en grains sont comparables (tabl. 2) ; or, pour les mêmes caractères, les liaisons entre les résidus ne sont pas non plus significatives.

\section{Relations entre les valeurs propres des lignées et leurs effets génétiques}

Pour les caractères où la variance d'A.G.C. est supérieure à la variance d'A.S.C. et malgré la faible préci- sion sur les estimations, les corrélations entre la valeur propre de la lignée $\left(P_{i}\right)$ et son A.G.C. (A.G.C. $)$ ont été estimées.

A l'exception du nombre de grains par silique sur les ramifications secondaires, les corrélations entre les estimations des A.G.C. et des valeurs propres des lignées sont toutes hautement significatives et plus ou moins élevées $(0,51$ à 0,83$)$ selon les caractères (voir tabl. 3). Hormis celles du rendement, les valeurs obtenues en 1981 sont légèrement supérieures à celles de 1979, mais la supériorité n'est jamais significative. Pour tous les caractères conditionnés essentiellement par $\hat{\gamma}_{\mathrm{g}}$, il existe donc une liaison positive élevée entre la valeur propre de la lignée et son A.G.C.

\section{DISCUSSION}

\section{A. Quelles orientations choisir pour la sélection de variétés hybrides?}

\section{Utilisation des informations sur la structure de la variabilité génétique}

Il ressort, pour la majorité des caractères étudiés, l'importance de la variance de l'A.G.C. dans l'expres-

TABLEAU 2

Corrélations entre les A.G.C. des géniteurs pour les différents caractères dans les essais de 1979 (a) et 1981 (b). Correlation between G.C.A. effects of selfed lines for the different characters and for both years, 1979 (a) and 1981 (b).

\begin{tabular}{|c|c|c|c|c|c|c|c|c|c|}
\hline Caractère & & $\mathrm{CF}$ & RENDT & P1000 & VERSE & NG/SIL.RI & NG/SIL.RII & NG/SIL & $\mathrm{PG} / \mathrm{SIL}$ \\
\hline \multirow[t]{2}{*}{ DF } & (a) & 0,15 & $-0,02$ & $0,50^{* *}$ & 0,34 & 0,16 & 0,18 & - & - \\
\hline & (b) & 0,27 & $-0,07$ & 0,08 & - & - & - & 0,22 & 0,20 \\
\hline \multirow[t]{2}{*}{$\mathrm{CF}$} & (a) & & $0,79 * *$ & $-0,04$ & $-0,47 * *$ & $0,38^{* *}$ & $-0,07$ & - & - \\
\hline & (b) & & $0,72^{* *}$ & 0,04 & - & - & - & $0,42^{*}$ & $0,44^{*}$ \\
\hline \multirow[t]{2}{*}{ RENDT } & (a) & & & $-0,16$ & $-0,46^{* *}$ & $0,56 * *$ & 0,17 & - & - \\
\hline & (b) & & & $-0,17$ & - & - & - & 0,21 & 0,31 \\
\hline \multirow[t]{2}{*}{ P1000 } & (a) & & & & $-0,38$ & $-0,36^{*}$ & $-0,31$ & - & - \\
\hline & (b) & & & & - & - & - & 0,17 & 0,22 \\
\hline \multirow[t]{2}{*}{ VERSE } & (a) & & & & & $-0,21$ & 0,29 & - & - \\
\hline & (b) & & & & & - & - & - & - \\
\hline \multirow[t]{2}{*}{ NG/SIL.RI } & (a) & & & & & & 0,27 & - & - \\
\hline & (b) & & & & & & - & - & - \\
\hline \multirow[t]{2}{*}{ NG/SIL.RI } & (a) & & & & & & & - & - \\
\hline & (b) & & & & & & & - & - \\
\hline \multirow[t]{2}{*}{ NG/SIL } & (a) & & & & & & & & - \\
\hline & (b) & & & & & & & & $0,85^{* *}$ \\
\hline
\end{tabular}

* Significatif avec un risque de $5 \%$.

** Significatif avec un risque de $1 \%$.

* Significant with a $5 \%$ risk.

** Significant with a $1 \%$ risk.

TABLEAU 3

Estimées des corrélations entre l'A.G.C. et la valeur propre d'une lignée pour chaque caractère dans les essais de 1979 (a) et 1981 (b). Estimates of the correlations between the G.C.A. effects and the own values of selfed lines for each character and for both years, 1979 (a), 1981 (b).

\begin{tabular}{|c|c|c|c|c|c|c|c|c|c|}
\hline Caractère & DF & $\mathrm{CF}$ & RENDT & P1000 & VERSE & NG/SIL.RI & NG/SIL.RII & NG/SIL & $\mathrm{PG} / \mathrm{SIL}$ \\
\hline (a) & $0,64 * *$ & $0,52^{* *}$ & $0,78^{* *}$ & $0,56^{* *}$ & $0,71 * *$ & $0,67 * *$ & 0,30 & - & - \\
\hline (b) & $0,83^{* *}$ & $0,65^{* *}$ & $0,73^{* *}$ & $0,81 * *$ & - & - & - & $0,70^{* *}$ & $0,72^{* *}$ \\
\hline
\end{tabular}

** Significatif au seuil de $1 \%$.

** Significant with a $1 \%$ risk. 
sion de la variabilité génétique totale. Une grande partie de la variance génétique est «prévisible» dans les descendances. La sélection pour la valeur hybride portera donc sur les A.G.C. des lignées avant même la phase de production de l'hybride, et nécessitera le test d'un grand nombre de géniteurs de la population. Elle sera d'autant plus efficace qu'elle tiendra compte de la valeur propre des lignées, paramètre corrélé positivement à leur A.G.C.

L'ensemble de ces résultats n'a de sens que pour la population de lignées de colza sélectionnées.

\section{Constitution de pools génétiques}

Chez le colza, la production de semences hybrides implique l'utilisation d'un système de stérilité mâle. Dans le cas d'un système fonctionnel, on peut envisager la sélection de 2 groupes variétaux, le $1^{\text {er }}$ constitué de variétés mâle-stériles et mainteneuses de stérilité et le $2^{\mathrm{e}}$ de variétés restauratrices de fertilité. On pourrait définir un ensemble de testeurs à base large dans chaque groupe. Les variétés mâle-stériles seraient alors testées vis-à-vis de l'ensemble des testeurs restaurateurs de fertilité et inversement. Les tests de descendances se feraient sur des demi-frères puisque la variance d'A.G.C. est élevée. Les meilleures lignées sélectionnées dans les 2 groupes seraient ensuite intercroisées pour la production d'hybrides commerciaux. Parallèlement, le matériel génétique de chacun des 2 groupes pourrait être amélioré par sélection récurrente réciproque. Dans chaque groupe, il sera inclus une grande diversité génétique, en particulier du matériel européen et/ou asiatique dont la complémentation génique apparaît très favorable (LEFORT-BUSON \& DATTÉE, 1985). Cependant, il est aussi nécessaire de prendre en compte les contraintes liées à la qualité de la graine, les variétés hybrides devant être sans acide érucique et à faible teneur en glucosinolates. Les difficultés résident alors dans la création de 2 populations variables au plan génétique et complémentaires, l'une mâle-stérile et l'autre restauratrice de fertilité.

\section{Incidences des contraintes liées à la production de semences}

Actuellement, aucun système de stérilité mâle n'est encore fonctionnel. De plus, la diversité génétique au sein du matériel mâle-stérile et restaurateur de fertilité est faible : les plantes en cours de conversion ne sont issues que de quelques lignées de valeur propre élevée. Compte tenu des contraintes matérielles des programmes de conversion, leur développement ne peut être que très limité au moins pour le groupe des mâlestériles ; l'introduction d'un ou plusieurs gènes de restauration dans le groupe des restaurateurs présente moins de contraintes.

Il s'avère donc nécessaire d'introduire des lignées ayant déjà une bonne A.G.C. dans les schémas de conversion. La difficulté est justement d'estimer avec précision l'A.G.C. du fait des difficultés matérielles liées à la production manuelle de semences hybrides. En dehors de l'information fournie par un index combinant l'A.G.C. et la valeur propre des géniteurs, nous envisageons de rechercher d'autres prédicteurs de la valeur hybride dans la population parentale afin d'aug- menter la précision du jugement et la valeur des lignées.

\section{Sélection combinée}

La sélection portera sur la productivité et les facteurs de sa régularité dont, entre autres, les résistances à la verse et à divers parasites.

L'efficacité de la sélection peut être renforcée par l'utilisation de l'information relative à différents caractères liés au rendement, et ce d'autant plus que l'héritabilité de ces caractères sera plus élevée. Les estimations des corrélations entre le rendement, d'une part, et le couvert foliaire au début floraison ou le poids de grains par silique, d'autre part, sont respectivement élevées $(\hat{\rho} \# 0,75)$ et moyennes ( $\hat{\rho} \# 0,44)$, mais l'héritabilité de ces 2 caractères est faible $\left(\mathrm{h}^{2} \# 0,25\right)$, de l'ordre de celle du rendement. Les caractères dont l'héritabilité est plus élevée, la date de début floraison et le poids de 1000 grains, ne sont par contre pas corrélés au rendement au niveau génétique.

Pour les caractères dont la liaison avec le rendement est moyenne ou élevée, comme pour le rendement, un effort doit être fait pour diminuer la variance liée à l'environnement et, par conséquent, augmenter la précision des estimations de l'héritabilité.

\section{B. Analyse de l'hétérosis}

Dans la $1^{\text {re }}$ partie, nous avions montré que l'hétérosis se manifeste, pour la plupart des caractères étudiés, au cours du développement végétatif et à maturité. La comparaison des hybrides $\mathrm{F} 1$ et des lignées a révélé :

- des corrélations entre effets résiduels du même ordre de grandeur pour les divers caractères ;

- une variabilité génétique comparable dans les 2 populations;

- une continuité entre les distributions des lignées et des hybrides.

Nous concluons ici à une prépondérance de la variance d'A.G.C. dans la variabilité génétique. De plus, les effets d'A.G.C. sont reliés positivement aux valeurs propres des lignées, même pour le caractère « hauteur au début floraison » pour lequel les effets spécifiques sont significatifs et élevés.

La cohérence entre ces résultats suggère qu'il n'y a pas de différence profonde entre l'expression d'un caractère chez les hybrides et chez les lignées. Ainsi, l'hétérosis serait dû à une accumulation de gènes ou de linkats non allèles favorables à l'expression des divers caractères ; les linkats ont été définis par DEMARLY (1968) comme des blocs de gènes coadaptés résultant d'un processus de sélection naturelle ou induite. La faible importance des effets d'A.S.C. peut être interprétée comme une prédominance des effets d'épistasie cis. Ceux-ci, dans une structure génétique hybride seraient directement intégrés dans les linkats préexistants chez les lignées. On pourrait ainsi expliquer l'existence simultanée d'un hétérosis élevé et d'une variance d'A.G.C. importante en valeur relative.

L'accumulation de gènes ou groupes de gènes dominants pour lesquels il y a une bonne complémentarité de fonctionnement se traduirait par une meilleure efficacité et une plus grande rapidité des processus de dif- 
férenciation et de développement de la plante. Les hybrides ont en effet une implantation plus rapide et sont plus vigoureux que les lignées tout au long de leur croissance ; à la sortie de l'hiver la reprise en végétation est aussi plus rapide (LEFORT-BUSON, données non publiées). Par ailleurs, la supériorité du poids des parties vertes des plantes hybrides comparée aux lignées aux stades «rosette » et «début floraison 》 serait davantage due à un accroissement de la taille des feuilles et non de leur nombre (LEFORT-BUSON \& DATTEE, 1982a). Ceci est un argument supplémentaire en faveur d'un meilleur tonctionnement physiologique des structures hybrides sans toutefois qu'existe une différence spécifique entre homozygotes et hétérozygotes.

La diversité de l'information génétique des hybrides peut leur conférer une meilleure homéostase comme l'a souligné LERNER (1958). Leur moindre sensibilité aux variations de l'environnement révélée par une variance résiduelle globalement plus faible que celle des lignées et un effet d'hétérosis nettement plus marqué en conditions difficiles peut d'ailleurs être un argument en faveur de cette hypothèse. La confirmation d'une plus grande « stabilité » des hybrides, sur plusieurs années ou même plusieurs lieux, permettrait d'apporter des éléments supplémentaires dans la discussion concernant le choix du type variétal à créer.

Les arguments qui viennent d'être avancés conduisent à l'hypothèse d'une fixation possible de l'hétérosis à long terme, dans la mesure où le taux de recombinaison entre gènes (ou linkats) non allèles ne sont pas nuls. SEDCOLE (1980), dans une revue synthétique sur les différentes théories de l'hétérosis, arrive d'ailleurs aux mêmes conclusions à partir de nombreux exemples sur diverses espèces végétales.

Des résultats comparables (effet d'hétérosis et prépondérance de la variance additive) ont en effet déjà été mis en évidence chez le maïs (MOLL \& ROBINSON, 1967) chez quelques plantes fourragères (BREESE \& HAYWARD, 1972) et chez la luzerne (GALlAIS, 1977) ; l'hypothèse d'additivité de linkats a aussi été retenue pour expliquer une grande partie de l'hétérosis. L'importance de la vigueur hybride chez ces espèces allogames nous incite à penser que les phénomènes de complémentation épistatique (de type «trans ») entre linkats sont plus développés que chez les espèces autogames ou semi-autogames. Pour ces dernières, l'hypothèse de l'accumulation de facteurs favorables et de relation d'épistasie " cis » (DEMARLY, 1977) permet d'expliquer la plupart des résultats et est cohérente en particulier avec la liaison entre l'A.G.C. et la valeur propre d'une lignée.

Les résultats montrent enfin les variations des différents effets génétiques en fonction du caractère ou même de l'année. Le rapport de la variance d'A.G.C. à celle d'A.S.C. est généralement plus élevé en 1979 qu'en 1981 où les conditions climatiques ont été beaucoup plus dures ; la portée de cette affirmation reste limitée car les effets d'A.S.C. sont peu ou non significatifs. Il est clair toutefois que la variance d'A.G.C. reste prépondérante dans l'expression de la variabilité totale. En conditions difficiles, il y aurait donc de légères modifications de nature des effets génétiques s'accompagnant d'une contribution plus élevée des interactions entre linkats.

Selon GALLAIS (1977), la formation des linkats au cours des cycles de sélection aurait pu conduire à une intégration progressive des effets d'épistasie entre loci à l'intérieur de ces linkats. Les caractères dont la valeur sélective est élevée, rendement et composantes, et dont les gènes sont "proches » dans le génome manifesteraient ainsi très peu d'effets épistasiques. Par contre, pour les caractères à valeur sélective faible, hauteur et peuplement à maturité, dont les gènes seraient plus dispersés dans le génome, des effets d'épistasie ont été mis en évidence (LEFORT-BUSON \& DATTEE, $1982 b$ ). On peut penser que la sélection naturelle ou artificielle renforce la cohésion des structures géniques gouvernant des caractères à forte valeur sélective et, au contraire, favorise des réorganisations entre gènes gouvernant des caractères de valeur sélective moindre. Le processus étant continu, on peut supposer aussi que les réorganisations particulièrement favorables sont à leur tour protégées des recombinaisons et conduisent alors à de nouveaux linkats.

\section{CONCLUSION}

Les résultats obtenus, de par l'échantillonnage pratiqué et du fait de leur homogénéité sur 2 ans, ont permis de dégager quelques points relatifs à l'organisation de la variabilité génétique dans la population de lignées de colza oléagineux et de comparer cette organisation à celle de diverses espèces cultivées.

Chez le colza oléagineux, l'importance de l'hétérosis conduit à souligner l'intérêt général de la structure hybride Fl comme structure variétale, dans l'hypothèse d'obtention d'un système opérationnel de contrôle de la pollinisation. Il reste cependant nécessaire de contrôler la stabilité du gain dans diverses conditions environnementales et d'étudier les résistances aux maladies des structures hybrides.

Les modalités de sélection peuvent s'envisager sous un aspect classique de sélection récurrente réciproque en profitant de complémentarités déjà existantes ou en créant 2 pools complémentaires. Cependant, la sélection ne pourra véritablement débuter qu'après transfert dans chaque population des systèmes de stérilité mâle et de restauration de la fertilité.

Sur le plan de l'expression génétique, il a souvent été mis en évidence une prédominance de la variance d'A.G.C. dans la variance génétique globale. Nous avons ici révélé, chez une espèce semi-autogame, un effet d'hétérosis important, une variance d'A.G.C. élevée et une corrélation positive entre la valeur propre de la lignée et son A.G.C. Ce résultat est à mettre en parallèle avec celui couramment observé chez des espèces allogames où coexistent une forte variance d'A.G.C. et une importante dépression de consanguinité. Une interprétation des faits peut être donnée en termes de structuration génétique sous forme de linkats ; cette interprétation est cohérente avec le résultat de similitude des variances génétiques dans les populations homozygote et hétérozygote.

Néanmoins, au plan biologique, nous nous heurtons ici à une limite des modèles statistiques de la génétique qui peuvent être utilisés comme de bons outils de prédiction mais qui restent peu explicatifs des mécanismes en jeu.

Reçu le 7 mai 1984.

Accepté le 16 octobre 1984 


\section{REMERCIEMENTS}

Nous remercions très sincèrement l'ensemble des techniciens du Laboratoire Colza de la Station d'Amélioration des Plantes de Rennes-Le Rheu pour leur assistance tout au long de ce travail.

\section{RÉFÉRENCES BIBLIOGRAPHIQUES}

Breese E. L., Hayward M. D., 1972. The genetic basis of present breeding methods in forage crops. Euphytica, 21, 324-336.

Buson Marianne, 1979. Hétérosis et paramètres génétiques de quelques caractères agronomiques chez le colza oléagineux (Brassica napus $L$.). These $3^{e}$ cycle, Paris VI, $78 \mathrm{p}$.

Demarly Y., 1968. La sélection des variétés synthétiques. Proc. 5th Eucarpia Congr. Milan, 269-295.

Demarly Y., 1977. Génétique et Amélioration des Plantes. Masson, France, $287 \mathrm{p}$.

Gallais A., 1977. Contribution à l'étude théorique et expérimentale de l'hétérosis chez une plante allogame autotétraploide. Thèse Doct. d'état, Paris VI, 306 p.

Hayman B. I., 1954. L'analyse de variance des tables diallèles. Biometrics, 10, 235-246.

Lefort G., 1977. Remarques sur la modélisation de l'interprétation des dispositifs diallèles. Ann. Amélior. Plant., 27 (2), 171-202.
Lefort-Buson Marianne, Dattée Yvette, 1982a. Genetic study of some agronomic characters in winter oilseed rape (Brassica napus L.). I. - Heterosis. Agronomie, 2 (4), 19-30.

Lefort-Buson Marianne, Dattée Yvette, 1982b. Genetic study of some agronomic characters in winter oilseed rape (Brassica napus L.). II. - Genetic parameters. Agronomie, 2 (4), 31-45.

Lefort-Buson Marianne, Dattée Yvette, 1985. Etude de l'hétérosis chez le colza oléagineux d'hiver (Brassica napus L.). I. - Comparaison de deux populations, l'une homozygote et l'autre hétérozygote. Agronomie, 5 (2), 101-110.

Lerner I. M., 1958. The Genetic Basis of Selection. John Wiley Sons, New York, 298 p.

Moll E. H., Robinson H. F., 1967. Quantitative genetic investigation of yield of maize. Züchter, 57, 192-199.

Sedcole J. R., 1980. A review of the theories of heterosis. Egypt. J. Genet. Cytol., 10 (2), 117-146. 